\title{
Supporting Kids' Active Travel during the Pandemic: Milwaukee SRTS Program
}

\author{
Marissa Meyer ${ }^{1}$, Nancy Pullen-Seufert ${ }^{2}$, and Michael Anderson ${ }^{3}$ \\ ${ }^{1}$ City of Milwaukee Department of Public Works, Milwaukee, U.S.A. \\ ${ }^{2}$ University of North Carolina Highway Safety Research Center, U.S.A. \\ ${ }^{3}$ Wisconsin Bike Fed, Milwaukee, U.S.A.
}

\begin{abstract}
The Milwaukee Safe Routes to School (SRTS) Program, a partnership between the city of Milwaukee Department of Public Works and the Wisconsin Bike Federation continued their commitment to children's active travel during the COVID-19 pandemic through offering "walk audit"-style traffic safety community walks and a variety of other biking and walking programs in neighborhoods with majority Black or Latine residents. These efforts included public involvement activities to discuss and incorporate preferences for planned infrastructure improvements; urban summer bicycle camps for kids; and support for teachers as SRTS champions. Staff made modifications such as conducting programming outdoors; modifying materials and facilitation methods to support physical distancing; providing face coverings and minimizing touching equipment. The National Center for Safe Routes to School recognized their efforts with the 2021 Vision Zero for Youth Innovation Award.
\end{abstract}

Keywords: walking, bicycling, child, built environment, stakeholder engagement 
Safe Routes to School (SRTS) programs aimed to make it safer for students to walk and bike to school and to increase the number of students who use these travel modes for the school commute. Together, the City of Milwaukee Department of Public Works (DPW) and the Wisconsin Bike Fed (Bike Fed) implemented the Milwaukee SRTS program. These two partners continued to support opportunities for walking and biking even while the COVID-19 pandemic forced schools to close and required widespread implementation of physical distancing and masking measures. Within the SRTS program, DPW focused on building street infrastructure improvements to increase traffic safety near schools and conducting public engagement for each of their projects. Pre-pandemic, the Bike Fed, which has been active in Milwaukee Public Schools for sixteen years, implemented two core activities in support of SRTS - during the school year they taught walking and biking programming and conducted promotional activities, and during the summer, they held bike camps for students.

Milwaukee is a city of diverse neighborhoods, each with their own unique assets and community voices. However, the Milwaukee metropolitan area is considered one of the most segregated in the U.S. (Frey, 2018). More than onehalf (approximately 53\%) of city of Milwaukee residents identify as Black, Asian, or another race other than White. Among the city's population aged five and older, $8.6 \%$ have limited English proficiency, equal to the national rate, but much higher than the statewide rate of $3.2 \%$. Poverty rates vary by race and ethnicity. Among Black people in the city, $39.9 \%$ are living in poverty, compared to $31.8 \%$ of Hispanic people and $14.8 \%$ of non-Hispanic White people. Childhood poverty is also a major concern. In Milwaukee, $83 \%$ of students qualify for the Federal Free and Reduced Price Meal Program compared to $42 \%$ statewide and $52 \%$ nationally (City of Milwaukee, 2016).

The Centers for Disease Control and Prevention's Healthy People 2030 reflected the continued need to increase physical activity for children (U.S. Department of Health and Human Services, n.d.). One of the many ways that structural and systemic racism play a role in physical activity disparities is the fact that young people of color and those living in low income communities have less access to environmental features that support physical activity, including walking and biking (Gordon-Larsen et al., 2006). To help address these disparities, the Centers for Disease Control and Prevention recognized the importance of creating supportive environments for walking and biking to school for child health (Community Preventive Services Task Force, 2018). In addition to these long-term disparities, the COVID-19 pandemic led to a reduction in physical activity for many children (Bates et al., 2020). To support Milwaukee schoolchildren's physical activity and access to streets where they live during the COVID-19 pandemic, DPW and Bike Fed conducted three major SRTS activities throughout the city.

Schools were selected for these activities based on a combination of targeting neighborhoods and schools with the greatest disparities in measures like traffic safety and income level compared to the City as a whole and identifying pre-existing relationships that could be leveraged. The program gained community member input on proposed infrastructure improvements through SRTS design workshops and traffic safety community walks, fostered kids' bicycle riding skills and a value for exploring their city through summer bike camps, and grew their school-based network for active travel through SRTS champion activities.

\section{SRTS Design Workshops}

Prior to the pandemic in February 2020, the Milwaukee Department of Public Works was in the midst of holding SRTS community workshops with students, school staff, teachers, parents and other stakeholders to identify priorities for infrastructure safety improvements around eight schools serving kindergarten through grade eight. These design workshops were part of the public involvement process for a federal Transportation Alternatives Program grant project that included about $\$ 75,000$ in construction funds for each school. The schools in the project were selected based on a variety of factors, including safety considerations like the incidence of pedestrian crashes nearby, equity considerations like the percentage of students eligible for the Federal Free and Reduced Price Meal Program, and the demographic makeup of the surrounding neighborhoods.

The workshop participants collaborated in small teams to first discuss the traffic safety issues around their school and learn about options for street improvements. In the second part of the workshop, each team decided what street improvements they thought should be built with the available project budget to address the safety issues they had identified.

Staff from the Bike Fed attended the workshops and quickly realized that the format and content could be integrated into lessons that help meet grade-level learning standards to create a "Child Friendly Cities" curriculum about walking, biking, and traffic safety. With the overall goal of centering youth voices in the design process, Bike Fed staff worked with teachers and other school staff to have students practice reading, math, and mapping activities leading up to the design workshop, and then after the workshop to write persuasive essays about why their team's street design should be built. These activities were positive experiences for students and teachers alike and culminated in selected students presenting their essays to an audience of various adult community leaders including the local alderman. Teachers stated that they had never seen students so engaged in a school project before. A realworld outcome had helped heighten the relevance of the learning objectives. Additionally, the design maps each student team created were sent to DPW staff to be analyzed alongside the initial public involvement maps.

Unfortunately, halfway through these workshops the COVID-19 pandemic began, which led to all Milwaukee schools closing and the remaining workshops being canceled. While DPW staff determined how they could 
adapt their remaining public involvement to fit COVID-19 transmission prevention guidelines, the Bike Fed made similar plans to modify and continue their SRTS programming.

\section{SRTS Traffic Safety Community Walks}

As the 2020-2021 school year began, DPW staff decided to continue the public engagement for the SRTS infrastructure project by holding socially-distanced, "walk audit"-style traffic safety community walks around each school. In addition to meeting COVID-19 transmission prevention guidance, the community walks provided an opportunity to get students and other community members moving and out in their neighborhoods. However, without the tools of a traditional public meeting like presentation slides, poster boards, or large maps laid out on tables, DPW's primary challenge was how to communicate information to participants and collect feedback while outdoors and on the move.

Prior to the pandemic, DPW staff had some experience with open-ended community walk audits. However, at that stage in the infrastructure project, three specific design options had already been developed based on the earlier workshops and other online surveys, and staff's primary goal was to get feedback on the three options and learn which option each school community preferred. Therefore, staff needed to find a way to clearly communicate each design option-including explaining the different street improvement types, such as curb extensions or traffic circles - and keep the options distinct in the minds of participants.

To help address this challenge, staff created an informational handout. The handout was two pages (double-sided) in length and included a map of the school area, photos of the different types of street improvements, and a chart showing which improvements would be built at which locations for each of the three design options. Colorcoding the options in the chart helped participants keep track of which option was which. Along the community walk, staff and participants stopped at the major locations around each school where safety improvements were being considered in order to describe the options at that location and get feedback from the community. When possible, staff also used chalk spray paint to draw outlines of traffic circles, curb extensions, and other street improvements on the street itself to help participants visualize what the changes would look like. At the safest locations, students assisted with the chalk spray paint as well.

Overall, getting feedback from the participants on individual locations during the walk was as easy as or even easier than it would have been during a traditional community meeting, as everyone involved was observing the location in real time. However, even with the handouts and other tools, at the end of the walk it was still somewhat challenging for participants to review the options as a whole and make a final recommendation on which one they preferred. Generally, additional time to review and discuss the options was all the participants needed to make their decision. Finding ways to summarize each option more succinctly, such as "the one with a lot of speed humps and a traffic circle," was also helpful for participants.

The Bike Fed supported these community walks with both general staffing, such as checking in participants, and more community walk-specific assistance, such as explaining details to individual participants who arrived late and providing traffic safety lookouts anytime people were in the street.

In addition to being outdoors and social distancing, some of the specific techniques to prevent COVID-19 transmission included requiring mask usage, providing masks to anyone who did not have one, providing hand sanitizer, sanitizing items like pens and clipboards between users, and providing all written information and photos in the handouts to avoid groups clustering around poster boards.

Actions to make the community walks feel more open and welcoming to communities of color and non-native English speakers included scheduling the walks at the times most convenient for the school community, providing interpretation and translation for walks in Hispanic/Latine and Hmong communities, limiting the total length of the community walk to one hour in order to both reduce the time commitment and avoid fatiguing participants, and using non-technical language. Unfortunately, due to COVID-19 restrictions staff could not provide water or other refreshments to participants. Attendance at the community walks was generally the same or higher than expected based on previous public engagement efforts in the same neighborhoods and the circumstances of the pandemic, suggesting that these efforts did have a positive impact.

\section{Bike Camps}

Bike camps provide an opportunity for youth to learn bicycle handling skills and navigation, and to experience the joy and independence bicycling offers, throughout the summer of 2020, the Bike Fed continued its summer bicycle camps in partnership with Milwaukee Recreation, COA and Boys \& Girls Clubs. Five camp sessions enabled youth who might otherwise be indoors to be outdoors and physically active. The camps served Milwaukee Public School students in grades four to eight enrolled at Community Learning Centers. Community Learning Centers serve as neighborhood hubs for community services including provision of free meals for K-12 students. In typical summers, campers take at least one weekly field trip by bus, but due to bussing and COVID19-related restrictions, these were all cancelled. With rides of two to 15 miles in length, students gained the ability to move through city streets cooperatively while developing their leadership skills and geographic knowledge, including many campers making their first trip to the shore of Lake Michigan.

The team made several changes to accommodate COVID-19 precautions, including providing all instruction 
outside on the playground. Students received their own personal protective equipment, including a branded Bike Fed gaiter for outdoors, disposable masks for entering buildings for bathroom breaks, hand sanitizer to hang from their bicycle handlebars, a backpack with water bottle, and the camp curriculum that had been modified to a booklet so that worksheets could be carried and then completed in parks. Bikes, stored in a line, accompanied that student's own backpack and helmet so everything could be retrieved independently by students with limited contact from the Safe Routes instructors. Additionally, instructors stayed at one site rather than working at multiple sites in a day so that if someone had contracted COVID, contact-tracing would be more effective. No cases in staff or students were reported.

\section{SRTS Champions}

The Bike Fed's SRTS approach includes serving as instigators of active travel culture at schools and then institutionalizing that work with school staff so that it can continue to grow. To be responsive to the shift to virtual learning and loss of in-person interactions at schools, the team pivoted its work to support growing school staff as SRTS champions by offering one-on-one support to teachers interested in shifting more of their trips to active travel modes. Bike Fed staff visited interested teachers' homes to provide customized route planning, do basic bike maintenance and outfit them with supplies such as a bike lock. In some cases, these teachers then initiated activities with their students. For example, one teacher took students on weekend rides at parks. All of her students received donated bikes after the teacher posted a request on her neighborhood Facebook page. Another teacher champion worked with students to participate in a bicycle art contest. Based on content provided by the Bike Fed, students drew their own pictures of bikes and submitted them for a chance to receive a bike, lock and helmet. Three students received new bikes through the contest. Additionally, the Bike Fed supported the launch of monthly walking clubs in a limited number of locations to foster social time and physical activity for students while maintaining the need for COVID precautions by social distancing and being outdoors. One school's club proved to be a meaningful platform in which to engage the police department about alternative strategies for community safety other than enforcement. In all, 15 teachers at 11 schools participated.

\section{Results and Next Steps}

The SRTS activities conducted by DPW and the Bike Fed during the COVID-19 pandemic successfully reached a variety of community members. Between the workshops, surveys, and community walk audits, over 300 participants in diverse neighborhoods gave input on the SRTS infrastructure projects. Students, teachers, parents, and other community members were able to participate in each step of the design process, including identifying safety issues around each school, developing design alternatives, and making the final selection of the preferred alternative.
In 2021, the Milwaukee SRTS program received the Vision Zero for Youth Innovation Award from the National Center for Safe Routes to School for serving as a model of continued commitment to safe, active travel for children during the pandemic (National Center for Safe Routes to School, 2021). The program's focus on places where Black and Latine families live was considered particularly important given the impact of systemic racism on all facets of life, including health, as recognized by Milwaukee Common Council in July of 2019 (American Public Health Association, n.d.).

While overall these programs were regarded as successful by DPW, the Bike Fed, and the school communities, in retrospect one limitation was the lack of evaluation measures available. For the traffic safety community walks, it would have been valuable to better understand community member's experiences with the walks and related activities. In addition to recording participation numbers, in the future conducting a brief exit survey could be used to get more specific feedback on how to further improve. Weather was also a major limiting factor for many of these activities, with some events either having low attendance or having to be rescheduled or canceled. For the summer bike camps, understanding any changes in participant feelings of independence, safety and social connectedness would be valuable both for understanding achievements of the intervention and to attract additional funding.

Following the COVID-19 pandemic, DPW and the Bike Fed plan to implement an updated SRTS program pilot for the 2021-2022 school year. Using local funding, the two organizations will work with the teaching staff at one elementary school to integrate classroom activities and learning goals with the city's public involvement process throughout the school year. Like the previous SRTS infrastructure project, the improvements selected by the school community will be constructed following the planning process.

In this 2021-2022 program, activities like the design workshops and community walks will be remixed with new ideas to elevate youth voices, such as combining a lesson on voting with asking the students to select which option they prefer for street improvements around their school. Most of these activities and other public engagement events will be clustered in the fall so that design and engineering work can take place over the winter. In the springtime staff from both agencies will work with the schools to hold a public art event celebrating the upcoming street improvements, which will be built over the summer. DPW and the Bike Fed intend to develop this program into an annual project with multiple schools per year as staff gain experience and streamline the process.

In the future, thanks to experience gained during the COVID-19 pandemic, DPW staff intend to use public engagement activities like community walks on as many projects as possible because of their proven value in elevating interactions with community members in person and on the streets in question. Additionally, DPW staff are increasing engagement with school communities on all 
projects - not just SRTS-specific projects - in order to get more input from the youngest members of the community.

Similarly, due to the growing popularity of the walking club concept begun during the 2020-2021 school year, the Bike Fed plans to use their stories to promote and expand this concept into the next school year. The walking club model, while simple, has proven to be a very effective engagement strategy and building block to help build capacity and excitement for further SRTS efforts. Longterm tracking of summer bike camp participants could reveal if and how their experiences influence their travel mode selection as they grow. Given the transient nature of this population, however, creative means would be necessary to track the students and follow-up.

Milwaukee and other communities could benefit from understanding the replicability of its SRTS community engagement strategies in other cities. City leaders, public health and transportation professionals could all gain from additional strategies that support physical activity in ways that are welcoming and community-centered.

\section{Correspondence should be addressed to}

Nancy Pullen-Seufert

UNC Highway Safety Research Center

730 Martin Luther King Jr, Blvd, Suite 300

CB 3430

Chapel Hill, NC 27599-3430

pullen@hsrc.unc.edu
(919)-962-7419

D Marissa Meyer: 0000-0003-3288-996X

(D) Nancy Pullen-Seufert: 0000-0003-4957-3259

\section{Acknowledgments}

This work was supported by the Federal Highway Administration, Wisconsin Department of Transportation, and City of Milwaukee under a Transportation Alternatives Program project ID 2984-13-03; Wisconsin Department of Transportation and Milwaukee Public Schools under Transportation Alternatives Program project ID 1009-0085.

\section{Conflict of Interest}

The authors do not have any conflicts of interest to declare.

\section{Author Contributions}

Conceptualization, M.M. and M.A.; Implementation, M.M. and M.A.; Writing - Original Draft, M.M. and N.P., Writing - Reviewing and Editing, M.M., N.P., and M.A.

\section{Creative Commons License:}

This work is licensed under a Creative Commons

Attribution-Noncommercial 4.0 International License (CC

BY-NC 4.0).

\section{References}

American Public Health Association. (n.d.). Racism is a Public Health Crisis. Retrieved May 24, 2021 from https://www.apha.org/topics-and-issues/health-equity/racism-and-health/racism-declarations

Bates, L. C., Zieff, G., Stanford, K., Moore, J. B., Kerr, Z. Y., Hanson, E. D., Gibbs, B. B., Kline, C. E., \& Stoner, L. (2020). COVID-19 impact on behaviors across the 24-hour day in children and adolescents: Physical activity, sedentary behavior, and sleep. Children, 7, no. 9: 138. https://doi.org/10.3390/children7090138

City of Milwaukee: Community Health Assessment. (2016). City of Milwaukee Health Department.

Frey, W. (2018). Black-white segregation edges downward since 2000, census shows. Brookings. Retrieved May 24, 2021 from https://www.brookings.edu/blog/the-avenue/2018/12/17/black-white-segregation-edges-downward-since-2000-censusshows/

Gordon-Larsen, P., Nelson, M. C., Page, P., \& Popkin, B. M. (2006). Inequality in the built environment underlies key health disparities in physical activity and obesity. Pediatrics, 117(2), 417-424. https://doi.org/10.1542/peds.2005-0058 
Journal of Healthy Eating and Active Living

2021, Vol. 1, No. 4, pgs. 229-234

National Center for Safe Routes to School. (2021, April 5). Milwaukee receives Vision Zero for Youth Innovation Award, recognition for efforts to support safe walking and biking amid COVID-19 pandemic [Press release]. http://www.visionzeroforyouth.org/wp-content/uploads/2021/04/2021-VZY-Award-PR-Milwaukee-FINAL.pdf

Physical Activity: Interventions to Increase Active Travel to School. (2018). Community Preventive Services Task Force.

Retrieved from https://www.thecommunityguide.org/findings/physical-activity-interventions-increase-active-travel-

$\underline{\text { school }}$

U.S. Department of Health and Human Services. (n.d.). Increase the proportion of children who do enough aerobic physical activity - PA-09. Healthy People 2030. Retrieved May 24, 2021, from https://health.gov/healthypeople/objectives-anddata/browse-objectives/physical-activity/increase-proportion-children-who-do-enough-aerobic-physical-activity-pa-09 